\title{
Fonoaudiologia, gerontologia e a apneia obstrutiva do sono: relato de caso
}

\section{Speech-language pathology, gerontology and obstructive sleep}

\author{
apnea: case report
}

Danielle Barreto e Silva' (1), Camila de Castro Corrêa $a^{2,3}$ (1)

\section{RESUMO}

A eficácia da terapia fonoaudiológica está comprovada para a apneia obstrutiva do sono (AOS), principalmente para a população adulta. Entretanto, estudos com população acima de 65 anos são escassos. Assim, o objetivo do presente estudo foi analisar a evolução clínica longitudinal de um sujeito idoso com AOS submetido à terapia miofuncional orofacial (TMO). Caso clínico, sexo masculino, 72 anos, com AOS, submetido à TMO após ter realizado avaliação clínica, exames de polissonografia (PSG) e avaliação clínica otorrinolaringológica, bem como a aplicação da escala de sonolência de Epworth, a classificação de Mallampati e a avaliação fonoaudiológica miofuncional orofacial em três momentos distintos: antes da TMO, após a TMO e após 22 meses da TMO. A TMO se configurou na realização de exercícios isométricos e isotônicos para região orofacial e faríngea, treino funcional em sessões semanais de 30 minutos, durante cinco meses, quando, então, as sessões foram espaçadas. Após os 22 meses de TMO, observaramse evoluções nos parâmetros objetivos e subjetivos relativos ao sono, que foram analisados e identificados por meio de uma nova polissonografia (PSG), com os seguintes dados: exame basal, 24,5 eventos/hora e último exame de 4,63 eventos/hora. Verificou-se acentuada melhora da sonolência diurna excessiva e, ainda, melhora dos parâmetros miofuncionais orofaciais. Deste modo, pôde-se constatar que a TMO demonstrou resultados eficientes e eficazes na intervenção e no tratamento terapêutico fonoaudiológico, tendo como base a AOS do paciente idoso. Ressalta-se que tais resultados foram acompanhados e controlados por meio de avaliação multidisciplinar de modo longitudinal.

Palavras-chave: Fonoaudiologia; Apneia obstrutiva do sono; Sono; Idoso; Sistema estomatognático

\begin{abstract}
The effectiveness of speech-language therapy is proven for obstructive sleep apnea (OSA), especially for the adult population. However, studies with a population over 65 years are scarce. Thus, the aim of the present study was to analyze the longitudinal clinical evolution of an OSA elderly subject undergoing Orofacial Myofunctional Therapy (OMT). Male clinical case, 72 years old, with OSA submitted to OMT, polysomnography (PSG) exams, otorhinolaryngological clinical evaluation, Epworth sleepiness scale application, Mallampati classification and orofacial myofunctional speech evaluation in 3 differents moments (before OMT, after OMT and after 22 months of OMT). OMT was configured to perform isometric and isotonic exercises for the orofacial and pharyngeal regions and functional training in weekly sessions of 30 minutes for 5 months. After that period, the sessions were spaced for biweekly, monthly and quarterly. After 22 months of OMT, an evolution was observed in the objective and subjective parameters related to sleep, identified by polysomnography (baseline test: 24.5 events / hour and last test of 4.63 events per hour), improvement in excessive daytime sleepiness and improvement of myofunctional orofacial parameters. Thus, it was observed that OMT demonstrated efficient and effective results of speech therapy intervention and therapeutic treatment based on OSA in this case report of an elderly person. It is noteworthy that such results were monitored and controlled through multidisciplinary evaluation in a longitudinal way.
\end{abstract}

Keywords: Speech, Language and Hearing Science; Obstructive sleep apnea; Sleep; Aged; Stomatognathic system

\footnotetext{
Trabalho realizado na Universidade de Brasília - UnB - Brasília (DF), Brasil.

${ }^{1}$ Secretaria de Estado de Educação - Brasília (DF), Brasil.

${ }^{2}$ Faculdade de Ceilândia, Universidade de Brasília - Brasília (DF), Brasil.

${ }^{3}$ Centro Universitário Planalto do Distrito Federal - UNIPLAN - Brasília (DF), Brasil.

Conflito de interesses: Não.

Contribuição dos autores: DBS e CCC contribuíram para a estruturação do trabalho, coleta, tabulação, análise e interpretação dos dados, redação, revisão do artigo e consentimento da versão final para publicação.

Financiamento: Nada a declarar.

Autor correspondente: Danielle Barreto e Silva. E-mail: danielle.pitta@hotmail.com

Recebido: Fevereiro 13, 2021; Aceito: Abril 08, 2021
} 


\section{INTRODUÇÃO}

O processo do envelhecimento implica alterações neurobiológicas estruturais, funcionais e químicas. É um processo natural do desenvolvimento humano, que pode acarretar deficit cognitivos, físicos e comportamentais, influenciados por fatores internos (envelhecimento das células que comprometem as respostas da função celular, radicais livres) ou externos (como uma alimentação balanceada e a prática de atividade física regular) ao organismo ${ }^{(1)}$.

A perda da musculatura esquelética devido à idade, denominada sarcopenia, é um dos sintomas que acomete, principalmente, indivíduos sedentários, mas também ocorre em indivíduos saudáveis e ativos, impactando em maior ou menor grau a realização de atividades de vida diária, podendo culminar na redução da autonomia. Diferentes fatores influenciam a sarcopenia, como alterações hormonais, perda de neurônios motores, nutrição inadequada, inatividade física e níveis de inflamação crônica ${ }^{(2)}$.

A diminuição de fibras musculares e da força muscular não se restringe aos grandes segmentos corporais, porém, as estruturas orofaciais e faríngeas também são influenciadas por tais alterações relacionadas ao envelhecimento. Exemplos desse panorama são a presbifonia, definida como alterações vocais dos músculos da laringe e pregas vocais, decorrentes do envelhecimento ${ }^{(3)}$, e a presbifagia, degeneração fisiológica do mecanismo da deglutição, devido ao envelhecimento sadio das fibras nervosas e musculares ${ }^{(4)}$. Assim, essa perda da força muscular orofacial e faríngea pode resultar em alteração das funções do sistema estomatognático, quais sejam, mastigação, deglutição, fala e respiração ${ }^{(3,4)}$.

Especificamente no que se refere à respiração e à apneia obstrutiva do sono (AOS), distúrbio respiratório que interfere na qualidade e quantidade do sono, que pode levar a possíveis deficit cognitivos decorrentes da hipóxia intermitente, alterações na arquitetura do sono e acúmulo de resíduos metabólicos ${ }^{(5)}$, verifica-se que a adaptação do tratamento Continuous Positive Airway Pressure (CPAP)/Pressão Positiva Contínua das Vias Aéreas proporciona melhora da sonolência diurna excessiva, diminuição da fadiga para realizar as atividades de vida diárias, dos sintomas depressivos e melhora na qualidade de $\operatorname{vida}^{(6)}$.

A atuação fonoaudiológica no tratamento dos distúrbios do sono vem se consolidando como uma das opções isoladas ou combinadas nos distúrbios respiratórios do sono, como na AOS, com métodos adequados para adultos ${ }^{(7)}$. Os efeitos da terapia fonoaudiológica miofuncional orofacial se relacionam à diminuição do índice de apneia e hipopneia (IAH), do índice de despertar, do relato de sonolência diurna excessiva, bem como ao aumento da qualidade do sono e da qualidade de $\operatorname{vida}^{(7)}$. Ainda se observa uma lacuna importante no que se refere à compreensão dos efeitos diretos na musculatura e, também, abrangendo a população geriátrica, o que é preocupante, devido à maior prevalência da AOS conforme o avanço da idade ${ }^{(8)}$.

Estudos identificam alterações nas fibras musculares dos tecidos da faringe em pacientes com $\mathrm{AOS}^{(9)}$. Comparado a indivíduos sem AOS, foi encontrada uma redução das fibras Tipo II, de contração rápida e alta fatigabilidade, no músculo constritor superior da faringe, podendo levar à redução da eficiência dessa musculatura e envolvimento na fisiopatogenia da doença. Somado a isso, foi verificada a presença de proteínas de colágeno tipo I, que estão mais presentes no envelhecimento, favorecendo a diminuição da elasticidade do tecido. Assim, o indivíduo idoso com AOS tende a apresentar maior prejuízo no mecanismo de manutenção da patência das vias aéreas superiores (VAS), diretamente dependente da sensibilidade e contratilidade da musculatura orofacial e faríngea ${ }^{(9)}$.

Assim, o objetivo deste estudo foi analisar a evolução clínica longitudinal de um sujeito idoso com AOS, submetido à terapia fonoaudiológica miofuncional orofacial.

\section{APRESENTAÇÃO DO CASO CLÍNICO}

O presente estudo foi aprovado pelo Comitê de Ética em Pesquisas em Seres Humanos da Universidade de Brasília - UnB, sob registro 37/2004. O participante assinou o Termo de Consentimento Livre e Esclarecido.

Sujeito do sexo masculino com 72 anos, índice de massa corpórea $(\mathrm{IMC})=28,3 \mathrm{~kg} / \mathrm{m} 2$, hipertenso, sob uso de medicamento hidrocloatiazida, maleato de anafranil, pressão arterial (PA) com variação entre 16x10 e 14x9; apresentou queixa inicial de roncos leves, segundo o paciente, porém, o relato da esposa foi que eram intensos e que chegavam a acordá-la durante a noite; sonolência excessiva diurna (SED), cochilos frequentes ao longo do dia e referência de fadiga ao acordar; dificuldade de concentração e memória que impactava a qualidade de vida e o desempenho de atividades da vida diária.

O paciente apresentava diagnóstico prévio de AOS moderada e, pelo exame de polissonografia tipo 1, houve a indicação do tratamento por meio do CPAP (em titulação, indicado o uso da pressão de $7 \mathrm{~cm}$ de $\mathrm{H}^{2} \mathrm{O}$ ). Entretanto, por questões financeiras e dificuldade de adaptação, a terapia por meio do CPAP foi comprometida. Iniciou acompanhamento fonoaudiológico, mediante orientação médica. Não foram evidenciadas alterações na avaliação otorrinolaringológica e no exame de nasofibrolaringoscopia.

\section{Instrumentos de avaliação}

Para mensuração dos resultados da TMO, foram adotados parâmetros controlados em três momentos: antes do início da TMO, com cinco meses de TMO e após 22 meses do início da TMO.

\section{Avaliação objetiva do sono}

Para avaliação objetiva do sono, foi realizada polissonografia tipo I, equipamento Alice 3 v1.20 CHealthdyne. Foram analisados e considerados os seguintes parâmetros ${ }^{(10)}$ :

- IAH - índice de apneia e hipopneia por hora de sono: leve - cinco a 15 apneias/hora; moderada - 15 a 30 apneias/hora; grave - acima de 30 apneias/hora ${ }^{(10)}$;

- IMD - índice de microdespertares: número de eventos de despertares, maior que três segundos e menor que 15 segundos, por hora de sono;

- Arquitetura do sono: distribuição dos estágios de sono NREM (non-rapid eye movement) e REM (rapid eye movement), ao longo da noite; 
- Porcentagem do sono REM: ocupa de 20\% a 25\% do tempo total de sono;

- Saturação média de oxihemoglobina: saturação de oxigênio no sangue;

- T90 (tempo abaixo de 90\% de saturação de oxihemoglobina).

\section{Escala de Sonolência de Epworth (ESE)}

Trata-se de um questionário autoaplicável que mensura as chances de sonolência diurna excessiva durante oito situações, em atividades diárias. $\mathrm{O}$ escore global varia de 0 a 24 , sendo que acima de 10 é indicativo de sonolência diurna excessiva ${ }^{(11)}$.

\section{Avaliação miofuncional orofacial}

Foi utilizado o protocolo MBGR (Marchesan, BerrentinFelix, Genaro, Rehder) adaptado para casos de $\operatorname{AOS}^{(7)}$, que visa mensurar o desempenho dos músculos e funções orofaciais por meio do estabelecimento de escores. Quanto maior o escore, mais alterações encontradas na avaliação miofuncional orofacial.

Tal avaliação foi realizada por meio de observação da postura corporal (cabeça, região cervical, ombro, perfil da face); da postura habitual; da simetria; da morfologia e da mobilidade e do tônus das estruturas orofaciais (lábios, bochechas, músculo masseter e músculo temporal, palato duro, palato mole e língua).

Quanto à avaliação das funções do sistema estomatognático - respiração, mastigação, deglutição - a respiração foi avaliada a partir da observação dos movimentos tóraco-abdominais, a fim de identificar o modo e tipo respiratório, bem como a coordenação pneumofonoarticulatória. Foram consideradas, também, a contração indevida ou reduzida de determinados grupos musculares e as dores faciais à mastigação e/ou ao abaixamento de mandíbula (dor na articulação temporomandibular (ATM)).

Outro aspecto avaliado importante foi referente às ausências dentárias e utilização de próteses, aspectos que podem estar comprometidos em razão da estreita relação que apresentam com a musculatura da orofaringe. Quanto maior a pontuação no protocolo, maior o comprometimento das estruturas e funções.

Para a análise do teste de Mallampati modificado, foi solicitado ao sujeito que deglutisse e abrisse a boca, mantendo a língua relaxada. Neste momento, foi observado o aspecto das estruturas e se era possível a visualização da parede posterior da faringe, tonsilas palatinas, arcos palatinos da úvula (grau I), visibilidade completa/parcial da úvula e parcial de tonsilas e arcos (grau II), visibilidade mínima do palato mole e se não se via tonsilas e pilares cobertos pela base de língua (grau III), ou se estava com visibilidade apenas de palato duro (grau IV) ${ }^{(7)}$.

\section{Intervenção fonoaudiológica (TMO)}

Na primeira fase, a TMO foi realizada semanalmente, por 30 minutos e, em cada sessão, foram trabalhados entre seis e oito exercícios. Foram propostos exercícios para adequação da postura, mobilidade e força da musculatura orofacial e faríngea. As estruturas priorizadas foram aquelas relacionadas com a obstrução pelo colapso das vias aéreas superiores durante o sono: assoalho da cavidade oral; língua; músculos mastigatórios, bucinador, masseter, pterigoideo lateral e medial; palato mole e úvula; músculos supra-hioideos e infra-hioideos; musculatura faríngea e as funções de respiração, mastigação e deglutição(7) (Quadro 1).

O controle da adesão se deu por meio do relato do sujeito, que manteve uma narração positiva, além de análise da execução dos exercícios propostos a cada sessão. A orientação foi que continuasse realizando a TMO, diariamente, entre as sessões presenciais.

$\mathrm{Na} 4^{\mathrm{a}}$ fase, houve a realização de quatro a cinco exercícios, diariamente, sendo indicados exercícios para treino funcional das funções orofaciais, para força e mobilidade dos músculos orofaciais. $\mathrm{O}$ tempo médio gasto com a realização dos exercícios em casa era entre dez e 15 minutos. Foram realizadas 32 sessões presenciais ao longo dos 22 meses de acompanhamento.

Ressalta-se que a fonoaudióloga que realizou as avaliações foi a mesma responsável pela execução das terapias.

Os dados antropométricos e clínicos gerais deste caso foram monitorados para se constatar a origem das modificações, ou não, longitudinalmente, no tratamento fonoaudiológico. Assim, observou-se constância dos parâmetros, ressaltandose, principalmente, o IMC que pode influenciar positivamente ou negativamente o tratamento muscular e funcional da Fonoaudiologia (Tabela 1).

O exame de polissonografia expressou melhora significativa longitudinalmente, reforçando a sistematização do acompanhamento realizado para incentivar a continuidade dos exercícios diários pelo sujeito (Tabela 2). O sujeito compareceu a todas as 27 sessões de terapia fonoaudiológica, mostrando-se motivado com os resultados subjetivos de melhora do sono e qualidade de vida.

A avaliação miofuncional orofacial expressou considerável melhora nos músculos e funções orofaciais, reforçando, principalmente, a postura e mobilidade de língua, assim como o modo respiratório, a eficiência mastigatória e deglutição (Tabela 3).

\section{DISCUSSÃO}

A intervenção fonoaudiológica nos transtornos respiratórios do sono tem se mostrado uma alternativa de tratamento eficaz, por meio da adequação das estruturas e funções do sistema estomatognático. Entretanto, ao se compreender que a prevalência da AOS na população idosa é maior, e o seu aumento em tamanho populacional no mundo, se faz necessária a descrição das possibilidades e limitações da atuação fonoaudiológica especificamente para esses casos.

Nesse sentido, o caso explanado foi o de um sujeito idoso de 72 anos (inicialmente), o que se tornou inédito, por ainda não haver outros processos intervencionais fonoaudiológicos voltados ao sono, para comparações. Vale salientar que os resultados demonstrados apresentaram representatividade apenas para o caso clínico atendido neste estudo, sendo necessários novos estudos com uma população maior, em um desenho de ensaio clínico randomizado controlado, para que se estabeleçam as possibilidade e limitações da atuação fonoaudiológica com a população idosa em distúrbios respiratórios do sono.

Pôde-se estabelecer a fundamentação dessa atuação, por meio dos mecanismos envolvidos na patogênese da AOS e como alterações anatômicas, neuromusculares e distribuição central da gordura podem indicar esse aumento da prevalência em indivíduos idosos ${ }^{(1,2,8)}$. Ainda ilustrando essas alterações 
Quadro 1. Descrição dos objetivos terapêuticos, exercícios e o modo de realização durante a terapia miofuncional orofacial

\begin{tabular}{|c|c|c|c|}
\hline Sessões duração & Objetivo & Exercício executado & Modo/frequência de realização \\
\hline $1^{\text {a }}$ Fase & $\begin{array}{c}\text { Trabalhar mobilidade de } \mathrm{mm} \text { orbicular } \\
\text { dos lábios e risório. }\end{array}$ & Protrair e retrair lábios. & 4 séries de 5 a 10 repetições. \\
\hline 16 sessões & $\begin{array}{l}\text { Trabalhar mobilidade de mm } \\
\text { intrínseca e extrínseca da língua; mm } \\
\text { supra-hioidea e infra-hioidea. }\end{array}$ & Protrair e retrair a língua. & 4 séries de 5 a 10 repetições. \\
\hline \multirow[t]{10}{*}{ Durante 5 meses } & $\begin{array}{c}\text { Trabalhar mobilidade de mm intrínseca } \\
\text { da língua. }\end{array}$ & $\begin{array}{l}\text { Lateralizar ponta de língua nas } \\
\text { comissuras labiais internamente. }\end{array}$ & 4 séries de 5 a 10 repetições. \\
\hline & $\begin{array}{c}\text { Trabalhar mobilidade de mm intrínseca } \\
\text { e extrínseca da língua e mm } \\
\text { supra-hioidea. }\end{array}$ & $\begin{array}{l}\text { Varrer palato duro com a ponta de } \\
\text { língua. }\end{array}$ & 4 séries de 5 a 10 repetições. \\
\hline & $\begin{array}{c}\text { Trabalhar mobilidade de mm intrínseca } \\
\text { e extrínseca de língua e } \mathrm{mm} \\
\text { supra-hioidea. }\end{array}$ & Rotação de língua no vestíbulo oral. & $\begin{array}{l}4 \text { séries de } 5 \text { repetições sentido } \\
\text { horário e anti-horário. }\end{array}$ \\
\hline & $\begin{array}{l}\text { Trabalhar a tonicidade de } \mathrm{mm} \\
\text { intrínseca e extrínseca de língua e mm } \\
\text { supra-hioidea }\end{array}$ & $\begin{array}{l}\text { Sugar língua no palato e manter } \\
\text { contração estática. }\end{array}$ & $\begin{array}{l}4 \text { séries de } 10 \text { a } 20 \text { segundos em } \\
\text { contração estática. }\end{array}$ \\
\hline & $\begin{array}{l}\text { Trabalhar a mobilidade e tonicidade de } \\
\text { mm do véu palatino e da úvula. }\end{array}$ & Articular $\mathrm{A} / \tilde{\mathrm{A}}$ & $\begin{array}{l}4 \times 20 \text { vezes, depois sustentar a } \\
\text { contração por até } 20 \text { segundos. }\end{array}$ \\
\hline & $\begin{array}{l}\text { Trabalhar a tonicidade, } \mathrm{mm} \text { faringe, } \\
\text { palato mole e bucinador, músculos } \\
\text { respiratórios. }\end{array}$ & Inspirar e soprar inflando bochechas. & 5 a 10 vezes. \\
\hline & Modo respiratório & $\begin{array}{l}\text { Lavagem com soro fisiológico e } \\
\text { alternância nasal. }\end{array}$ & $\begin{array}{c}\text { Essas orientações e treinos foram } \\
\text { inseridos em sequência ao trabalho } \\
\text { muscular correspondente. }\end{array}$ \\
\hline & Padrão mastigatório & $\begin{array}{l}\text { Mastigação bilateral sem contração } \\
\text { mm associada. }\end{array}$ & $\begin{array}{l}\text { Nesta fase, os exercícios foram } \\
\text { recomendados para serem realizados } \\
4 \times \text { todos os dias, em casa. }\end{array}$ \\
\hline & Deglutição & Sem contração mm associada. & \\
\hline & Fala & Amplitude articular. & \\
\hline \multicolumn{4}{|l|}{$2^{\mathrm{a}}$ Fase } \\
\hline 4 sessões & \multirow{3}{*}{\multicolumn{3}{|c|}{ Foram mantidos os exercícios na frequência de $4 \mathrm{x}$ ao dia. }} \\
\hline 2 meses & & & \\
\hline $3^{\text {a }}$ Fase & & & \\
\hline 6 sessões & \multirow{2}{*}{\multicolumn{3}{|c|}{ Foram mantidos os exercícios na frequência de $3 x$ ao dia. }} \\
\hline 6 meses & & & \\
\hline $4^{a}$ Fase & $\begin{array}{l}\text { Trabalhar mobilidade de mm intrínseca } \\
\text { e extrínseca da língua e mm supra- } \\
\text { hioidea e infra-hioidea. }\end{array}$ & Protrair e retrair a língua. & 4 séries de 5 a 10 repetições. \\
\hline 6 sessões & $\begin{array}{l}\text { Trabalhar mobilidade de mm intrínseca } \\
\text { e extrínseca da língua e mm supra- } \\
\text { hioidea. }\end{array}$ & $\begin{array}{l}\text { Varrer palato duro com a ponta de } \\
\text { língua. }\end{array}$ & 4 séries de 5 a 10 repetições. \\
\hline \multirow[t]{3}{*}{$\begin{array}{l}\text { No período de } 09 \\
\text { meses }\end{array}$} & $\begin{array}{l}\text { Trabalhar mobilidade de mm intrínseca } \\
\text { e extrínseca de língua e mm supra- } \\
\text { hioidea. }\end{array}$ & Rotação de língua no vestíbulo oral. & $\begin{array}{c}4 \text { séries de } 5 \text { repetições sentido } \\
\text { horário e anti-horário. }\end{array}$ \\
\hline & $\begin{array}{l}\text { Trabalhar a tonicidade de } \mathrm{mm} \\
\text { intrínseca e extrínseca de língua e mm } \\
\text { supra-hioidea. }\end{array}$ & $\begin{array}{l}\text { Sugar língua no palato e manter } \\
\text { contração estática. }\end{array}$ & $\begin{array}{l}4 \text { séries de } 10 \text { a } 20 \text { segundos em } \\
\text { contração estática. }\end{array}$ \\
\hline & $\begin{array}{l}\text { Trabalhar a mobilidade e tonicidade de } \\
\text { mm do véu palatino e da úvula. }\end{array}$ & Articular A/Ã. & $\begin{array}{l}4 \times 20 \text { vezes, depois sustentar a } \\
\text { contração por até } 20 \text { segundos. }\end{array}$ \\
\hline
\end{tabular}

Legenda: $\mathrm{mm}=$ musculatura

Tabela 1. Evolução clínica das queixas do caso de sexo masculino de 72 anos

\begin{tabular}{|c|c|c|c|}
\hline \multirow[b]{2}{*}{ Parâmetro } & $1^{a}$ Avaliação & $2^{\text {a }}$ Avaliação & $3^{a}$ Avaliação \\
\hline & Pré-TMO & $\begin{array}{l}\text { Pós-TMO } \\
\text { (5 meses) }\end{array}$ & $\begin{array}{c}\text { Pós-TMO } \\
\text { (22 meses) }\end{array}$ \\
\hline Idade (anos) & 72 & 72 & 74 \\
\hline ESE (pontos) & 13 & 1 & 1 \\
\hline $\begin{array}{c}\text { Pressão arterial } \\
(\mathrm{mmHq})\end{array}$ & $140 / 90$ & $120 / 80$ & $120 / 80$ \\
\hline IMC $\left(\mathrm{kg} / \mathrm{m}^{2}\right)$ & 28,3 & 28,6 & 28,5 \\
\hline
\end{tabular}

Legenda: TMO = terapia miofuncional orofacial; ESE = escala de sonolência de Epworth; $\mathrm{mmHg}=$ milímetros de mercúrio; IMC = índice de massa corporal; $\mathrm{kg} / \mathrm{m}^{2}=$ quilograma por metro quadrado neuromusculares, um estudo recente demonstrou diminuição da constrição faríngea durante a função de deglutição, provocando compensações, deglutições múltiplas e lentidão no trânsito do bolo entre fase oral e faríngea, caracterizando a presbifagia ${ }^{(12)}$. Transpondo esses dados, para o momento em que o sujeito está deitado, com a ação intensa da gravidade nessa região, espera-se, ainda mais, uma defasagem na amplitude da luz orofaríngea, tornando-se importante o trabalho muscular e funcional realizado pela Fonoaudiologia ${ }^{(7,13)}$.

Na primeira avaliação, o sujeito apresentou sonolência diurna excessiva além de hipertensão arterial sistêmica de difícil controle, o que é frequentemente encontrado em 
casos de $\operatorname{AOS}^{(1,5,7,11)}$. Quanto à sonolência diurna excessiva, observou-se melhora exponencial na avaliação imediata e na longitudinal, após a intervenção fonoaudiológica, confirmando a literatura $^{(7,13)}$, bem como, por meio do relato do paciente, houve um controle e manutenção da pressão arterial (PA) em valores de normalidade, após a TMO. Embora sem controle objetivo

Tabela 2. Evolução clínica dos parâmetros polissonográficos do caso de sexo masculino de 72 anos

\begin{tabular}{|c|c|c|c|}
\hline \multirow{2}{*}{$\begin{array}{l}\text { Parâmetro } \\
\text { PSG }\end{array}$} & $1^{\mathrm{a}}$ Avaliação & $2^{\text {a }}$ Avaliação & $3^{a}$ Avaliação \\
\hline & Pré-TMO & $\begin{array}{c}\text { Pós-TMO (5 } \\
\text { meses) }\end{array}$ & $\begin{array}{c}\text { Pós-TMO (22 } \\
\text { meses) }\end{array}$ \\
\hline $\mathrm{IAH}(\mathrm{e} / \mathrm{h})$ & 24,5 & 19,1 & 4,63 \\
\hline $\mathrm{SaO} 2 \mathrm{M}(\%)$ & 90 & 92 & 92 \\
\hline T90 (\%) & 7,8 & 1,9 & 4,6 \\
\hline № MD & 96 & 172 & 70 \\
\hline $\mathrm{MD}(\mathrm{e} / \mathrm{h})$ & 18,3 & 21,8 & 9,01 \\
\hline NREM2 (\%) & 65,6 & 56,2 & 52,4 \\
\hline REM (\%) & 13,9 & 14 & 22,2 \\
\hline
\end{tabular}

Legenda: $\mathrm{PSG}=$ polissonografia; $\mathrm{TMO}=$ terapia miofuncional orofacial; $\mathrm{IAH}=$ índice de apneia/hipopneia; e/h = eventos por hora; SaO2M = saturação de oxihemoglobina média; \% = percentual; T90 = tempo de saturação de oxigênio abaixo de $90 \% ; \mathrm{MD}$ = índice de microdespertares; $\mathrm{REM}=$ rapid eye movement; NREM2 $=$ Non-rapid eye movement fase 2 desse dado clínico, nem mudanças de hábitos que pudessem estar relacionados à questão, a TMO pode ter influenciado na melhora da permeabilidade aérea durante o sono, contribuindo para melhores parâmetros respiratórios, que, por sua vez, auxiliam no manejo das alterações cardiovasculares provocadas pela AOS; Quanto à hipertensão arterial sistêmica (HAS), o paciente começou a responder positivamente ao tratamento medicamentoso utilizado ${ }^{(14)}$. Ressalta-se, ainda, que não houve modificação do IMC, que poderia ser outro fator a interferir para essa relação direta ${ }^{(15)}$.

A melhora e manutenção longitudinalmente do desempenho muscular e funcional captadas pelo protocolo MBGR mostraramse de acordo com a literatura, que demonstra o impacto dessa modalidade de tratamento nos parâmetros polissonográficos ${ }^{(7,8,10)}$, também identificados no presente estudo. Esse efeito deve ser monitorado concomitantemente à manutenção do IMC, para não haver sobreposição de efeitos ${ }^{(15)}$, o que foi possível controlar nas avaliações deste caso, não havendo mudança significativa do IMC ao longo dos dois anos de acompanhamento.

Apesar de observada a melhora não apenas no protocolo MBGR, mas também no questionário subjetivo e na polissonografia, ressalta-se que ese foi um ponto de limitação do presente trabalho, não utilizando cegamento no processo de avaliação e aplicação da TMO. Sugere-se que novos estudos repliquem

Tabela 3. Evolução clínica da avaliação miofuncional orofacial do caso de sexo masculino de 72 anos

\begin{tabular}{|c|c|c|c|}
\hline & $1^{a}$ Avaliação & $2^{a}$ Avaliação & $3^{a}$ Avaliação \\
\hline & Pré-TMO & Pós-TMO (5 meses) & Pós-TMO (22 meses) \\
\hline Mallampati adaptado & 4 & 3 & 2 \\
\hline EXAME EXTRAORAL (melhor resultado $=0$ e pior $=30$ ) & 11 & 9 & 8 \\
\hline Face $($ melhor resultado $=0$ e pior $=15)$ & 6 & 6 & 6 \\
\hline Lábios $($ melhor resultado $=0$ e pior $=13$ ) & 3 & 2 & 2 \\
\hline Masseter (melhor resultado $=0$ e pior $=2$ ) & 2 & 1 & 0 \\
\hline EXAME INTRAORAL (melhor resultado $=0$ e pior $=61$ ) & 7 & 6 & 4 \\
\hline Lábios (melhor resultado $=0$ e pior $=5$ ) & 0 & 0 & 0 \\
\hline Bochechas $($ melhor resultado $=0$ e pior $=8$ ) & 2 & 2 & 2 \\
\hline Língua $($ melhor resultado $=0$ e pior $=20$ ) & 5 & 4 & $2^{*}$ \\
\hline Palato $($ melhor resultado $=0$ e pior $=8$ ) & 0 & 0 & 0 \\
\hline Tonsilas palatinas $($ melhor resultado $=0$ e pior $=4$ ) & 0 & 0 & 0 \\
\hline Dentes (melhor resultado $=0$ e pior $=5$ ) & \multicolumn{3}{|c|}{ prótese total fixa superior e inferior } \\
\hline Oclusão $($ melhor resultado $=0$ e pior $=11$ ) & 0 & 0 & 0 \\
\hline MOBILIDADE $($ melhor resultado $=0$ e pior $=68$ ) & 27 & 20 & $14^{*}$ \\
\hline Lábios $($ melhor resultado $=0$ e pior $=27$ ) & 11 & 8 & 6 \\
\hline Língua $($ melhor resultado = 0 e pior $=24$ ) & 12 & 10 & 6 \\
\hline Véu palatino $($ melhor resultado $=0$ e pior $=4$ ) & 2 & 1 & 1 \\
\hline Mandíbula (melhor resultado = 0 e pior $=13$ ) & 2 & 1 & 1 \\
\hline SENSIBILIDADE $($ melhor resultado $=0$ e pior $=65$ ) & 5 & 4 & 4 \\
\hline Tátil (melhor resultado $=0$ e pior $=55$ ) & 5 & 4 & 4 \\
\hline Dor à palpação (melhor resultado $=0$ e pior $=10$ ) & 0 & 0 & 0 \\
\hline TÔNUS (melhor resultado = 0 e pior = 6) & 4 & 1 & 1 \\
\hline Lábios (superior + inferior) (melhor resultado $=0$ e pior $=2$ ) & 1 & 0 & 0 \\
\hline Mento $($ melhor resultado $=0$ e pior $=1$ ) & 0 & 0 & 0 \\
\hline Língua $($ melhor resultado $=0$ e pior $=1$ ) & 1 & 1 & 1 \\
\hline Bochechas (direita + esquerda) $($ melhor resultado $=0$ e pior $=2$ ) & 2 & 0 & 0 \\
\hline FUNÇÕES OROFACIAIS (melhor resultado = 0 e pior $=123$ ) & 22 & 14 & $11^{*}$ \\
\hline Respiração (melhor resultado = 0 e pior $=5$ ) & 2 & 0 & 0 \\
\hline Mastigação $($ melhor resultado $=0$ e pior $=10$ ) & 4 & 2 & 1 \\
\hline Deglutição (melhor resultado $=0$ e pior $=36$ ) & 11 & 8 & 6 \\
\hline Fala $($ melhor resultado $=0$ e pior $=72$ ) & 5 & 4 & 4 \\
\hline
\end{tabular}

Legenda: TMO = terapia miofuncional orofacial; $\left({ }^{*}\right)$ o fonoaudiólogo notou evolução de postura de língua, aumento de mobilidade em lábios, língua, bochechas e palato mole, respiração nasal após início de TMO e melhor eficiência mastigatória e deglutição 
a metodologia, em uma casuística maior, em um processo de duplo cegamento.

A associação entre AOS, respiração oral ou mista, alterações nos padrões mastigatórios, vocais e de deglutição é comumente descrita na clínica fonoaudiológica direcionada ao sono $\mathrm{s}^{(3,7,13)}$. No caso deste estudo, foi possível confirmar esses achados, sendo observada a respiração de modo misto (oronasal), modificações funcionais da deglutição, com movimentos de projeção de cabeça, tensionamento de lábios, disfunção sensorial intraoral e redução da força da musculatura mastigatória e de língua, gerando prejuízo na eficiência do processo de mastigação.

$\mathrm{Na}$ Classificação de Mallampati, o presente caso apresentou, inicialmente, o grau IV, em que só é possível visualizar o palato duro. Esse achado ratifica a fisiopatologia envolvida no estreitamento e colapso das VAS nos casos de AOS, pelo desequilíbrio entre as forças constritoras e dilatadoras que mantêm a patência da luz faríngea ${ }^{(12)}$. No decorrer do acompanhamento longitudinal fonoaudiológico, também foi possível notar evolução desse parâmetro, similar a estudos anteriores ${ }^{(7,13)}$, observando melhora, principalmente, na comparação da última avaliação (grau II) com a primeira.

Assim como a melhora do Mallampati modificado, os efeitos positivos da TMO foram constatados com maior ênfase nos parâmetros polissonográficos, na avaliação após 22 meses: normalização do IAH, melhora da arquitetura e eficiência do sono, redução da fragmentação do sono, redução da intensidade e frequência dos roncos. Evidenciou-se evolução gradativa mais lentificada na AOS na população geriátrica. Portanto, para essa população, possivelmente a avaliação imediata após o término da TMO não deva ser priorizada, sugerindo-se aguardar um tempo maior de estabilidade dos resultados, concomitantemente aos acompanhamentos periódicos longitudinais. Esse acompanhamento longitudinal se fundamenta pela atual discussão sobre o comportamento progressivo dos mecanismos fisiológicos/ endótipos relacionados à AOS que interagem diretamente com a resposta imediata e mediata aos tratamentos elegidos ${ }^{(8)}$.

É preconizado, nos casos de AOS moderada e grave, o uso do CPAP como tratamento de primeira escolha. Segundo a literatura, idosos que usam CPAP apresentam melhor processamento cognitivo, memória e funções executivas, além da diminuição dos comprometimentos cardiovasculares e melhor qualidade de vida, uma vez que o CPAP reduz as paradas respiratórias, normaliza a ciclicidade dos estágios do sono, melhora a fragmentação do sono e reduz a queda da saturação da oxihemoglobina. No entanto, a aceitação (21\%) e a aderência (44\%) ao CPAP são reduzidas na população idosa ${ }^{(6)}$, fato comprovado no caso estudado. Assim, é necessário um planejamento terapêutico em conjunto com o médico responsável pelo diagnóstico e conduta e a equipe multidisciplinar, ressaltando, aqui, a participação do fonoaudiólogo e, principalmente, contando com a participação ativa do paciente, ponderando suas expectativas, condições socioeconômicas e fatores motivacionais.

A adesão efetiva à TMO é um dos pontos a serem considerados para evolução clínica satisfatória do indivíduo com AOS. Indivíduos idosos com AOS talvez tenham mais disponibilidade de tempo e empenho na prática diária dos exercícios propostos em longo prazo para que, assim, a musculatura, mesmo que mais lentamente, responda de forma positiva aos estímulos da TMO.

\section{COMENTÁRIOS FINAIS}

A intervenção fonoaudiológica da TMO gerou resultados positivos para este caso clínico específico de AOS moderada em um idoso que demonstrou boa adesão ao tratamento e, consequentemente, resultou longitudinalmente na melhora dos parâmetros miofuncionais e orofaciais, bem como nos achados polissonográficos. Ressalta-se, ainda, que a terceira avaliação apontou dados mais expressivos. Deste modo, sugere-se que novos estudos com enfoque nessa população específica sejam realizados, com o objetivo de elaborar procedimentos e protocolos padronizados para que a Fonoaudiologia venha a contribuir, cada vez mais, na avaliação e na intervenção desses pacientes.

\section{REFERÊNCIAS}

1. Liu-Ambrose T, Barha C, Falck RS. Active body, healthy brain: exercise for healthy cognitive aging. Int Rev Neurobiol. 2019;147:95-120. http://dx.doi.org/10.1016/bs.irn.2019.07.004. PMid:31607364.

2. Waltz TB, Fivenson EM, Morevati M, Li C, Becker KG, Bohr VA, et al. Sarcopenia, aging and prospective interventional strategies. Curr Med Chem. 2018;25(40):5588-96. http://dx.doi.org/10.2174/0929867324 666170801095850. PMid:28762310.

3. Vaca M, Mora E, Cobeta I. The aging voice: influence of respiratory and laryngeal changes. Otolaryngol Head Neck Surg. 2015 Set;153(3):40913. http://dx.doi.org/10.1177/0194599815592373. PMid:26156424.

4. Azzolino D, Damanti S, Bertagnoli L, Lucchi T, Cesari M. Sarcopenia and swallowing disorders in older people. Aging Clin Exp Res. 2019 Jun;31(6):799-805. http://dx.doi.org/10.1007/s40520-019-01128-3. PMid:30671866.

5. Gosselin N, Baril AA, Osorio RS, Kaminska M, Carrier J. Obstructive sleep apnea and the risk of cognitive decline in older adults. Am J Respir Crit Care Med. 2019;199(2):142-8. http://dx.doi.org/10.1164/ rccm.201801-0204PP. PMid:30113864.

6. Pallansch J, Li Y, Bena J, Wang L, Foldvary-Schaefer N. Patientreported outcomes in older adults with obstructive sleep apnea treated with continuous positive airway pressure therapy. J Clin Sleep Med. 2018;14(2):215-22. http://dx.doi.org/10.5664/jcsm.6936. PMid:29351819.

7. Ieto V, Kayamori F, Montes MI, Hirata RP, Gregório MG, Alencar AM, et al. Effects of oropharyngeal exercises on snoring: A randomized trial. Chest. 2015;148(3):683-91. http://dx.doi.org/10.1378/chest.14-2953. PMid:25950418.

8. Edwards BA, Redline S, Sands SA, Owens RL. More than the sum of the respiratory events: personalized medicine approaches for obstructive sleep apnea. Am J Respir Crit Care Med. 2019 Set 15;200(6):691-703. http://dx.doi.org/10.1164/rccm.201901-0014TR. PMid:31022356.

9. Duarte BB. Comparação histológica entre as fibras dos músculos palatofaríngeo e constritor superior da faringe em indivíduos com e sem apneia obstrutiva do sono [tese]. São Paulo: Universidade de São Paulo; 2017 [citado em $2021 \mathrm{Fev}$ 13]. Disponível em: http://www. teses.usp.br/teses/disponiveis/5/5143/tde-09082017-103210/

10. Malhotra RK, Kirsch DB, Kristo DA, Olson EJ, Aurora RN, Carden KA, et al. Polysomnography for obstructive sleep apnea should include arousal-based scoring: an american academy of sleep medicine position statement. J Clin Sleep Med. 2018;14(7):1245-7. http://dx.doi. org/10.5664/jcsm.7234. PMid:29991439. 
11. Bertolazi AN, Fagondes SC, Hoff LS, Pedro VD, Menna Barreto SS, Johns MW. Portuguese-language version of the Epworth sleepiness scale: validation for use in Brazil. J Bras Pneumol. 2009;35(9):877-83. http://dx.doi.org/10.1590/S1806-37132009000900009. PMid:19820814.

12. Mancopes R, Gandhi P, Smaoui S, Steele CM. Which physiological swallowing parameters change with healthy aging? OBM Geriatrics. 2021;5(1):16. http://dx.doi.org/10.21926/obm.geriatr.2101153.

13. Pitta D, Pessoa AF, Sampaio ALL, Rodrigues RN, Tavares MG, Tavares P. Oral myofunctional therapy applied on two cases of severe obstructive sleep apnea syndrome. Arq Int Otorrinolaringol. 2007;11:350-4.
14. Khattak HK, Hayat F, Pamboukian SV, Hahn HS, Schwartz BP, Stein PK. Obstructive sleep apnea in heart failure: review of prevalence, treatment with continuous positive airway pressure, and prognosis. Tex Heart Inst J. 2018;45(3):151-61. http://dx.doi.org/10.14503/ THIJ-15-5678. PMid:30072851.

15. Wang SH, Keenan BT, Wiemken A, Zang Y, Staley B, Sarwer DB, et al. Effect of weight loss on upper airway anatomy and the apnea-hypopnea index. The importance of tongue fat. Am J Respir Crit Care Med. 2020 Mar 15;201(6):718-27. http://dx.doi.org/10.1164/rccm.2019030692 OC. PMid:31918559. 